\title{
Structural Evolution of PCL during Melt Extrusion 3D Printing
}

DOI:

10.1002/mame.201700494

\section{Document Version}

Accepted author manuscript

Link to publication record in Manchester Research Explorer

\section{Citation for published version (APA):}

Mirihanage, W., Da Silva Bartolo, P. J., Poologasundarampilla, G., Hinduja, S., Vyas, C., Liu, F., \& Pape, I. (2018). Structural Evolution of PCL during Melt Extrusion 3D Printing. Macromolecular Materials and Engineering, 1700494-1700500. https://doi.org/10.1002/mame.201700494

\section{Published in:}

Macromolecular Materials and Engineering

\section{Citing this paper}

Please note that where the full-text provided on Manchester Research Explorer is the Author Accepted Manuscript or Proof version this may differ from the final Published version. If citing, it is advised that you check and use the publisher's definitive version.

\section{General rights}

Copyright and moral rights for the publications made accessible in the Research Explorer are retained by the authors and/or other copyright owners and it is a condition of accessing publications that users recognise and abide by the legal requirements associated with these rights.

\section{Takedown policy}

If you believe that this document breaches copyright please refer to the University of Manchester's Takedown Procedures [http://man.ac.uk/04Y6Bo] or contact uml.scholarlycommunications@manchester.ac.uk providing relevant details, so we can investigate your claim.

\section{OPEN ACCESS}




\section{Macromolecular Materials and Engineering STRUCTURAL EVOLUTION OF PCL DURING MELT EXTRUSION 3D PRINTING --Manuscript Draft--}

Manuscript Number:

Full Title:

Article Type:

Section/Category:

Keywords:

Corresponding Author:

Corresponding Author Secondary

Information:

Corresponding Author's Institution:

Corresponding Author's Secondary Institution:

First Author:

First Author Secondary Information:

Order of Authors:

Order of Authors Secondary Information:

Abstract:

Additional Information:

Question

Please submit a plain text version of your cover letter here.

Please note, if you are submitting a revision of your manuscript, there is an opportunity for you to provide your responses to the reviewers later; please
mame.201700494R2

STRUCTURAL EVOLUTION OF PCL DURING MELT EXTRUSION 3D PRINTING

Communication

Additive biomanufacturing; crystallization; in situ X-ray diffraction; screw-assisted melt extrusion.

Paulo Bartolo, Ph.D.

University of Manchester

Manchester, UNITED KINGDOM

University of Manchester

Fengyuan Liu

Fengyuan Liu

Cian Vyas

Gowsihan of Poologasundarampillai

lan Pape

Sri Hinduja

Wajira Mirihanage

Paulo Bartolo, Ph.D.

Screw-assisted material extrusion technique has been developed for tissue engineering applications to produce scaffolds with well-defined multi-scale microstructural features and tailorable mechanical properties. In this study, in situ timeresolved synchrotron diffraction was employed to probe extrusion-based 3D printing of polycaprolactone $(P C L)$ filaments. Time-resolved X-ray diffraction measurements revealed the progress of overall crystalline structural evolution of $P C L$ during $3 \mathrm{D}$ printing. Particularly, in situ experimental observations provide strong evidence for the development of strong directionality of PCL crystals during the extrusion driven process. Results also show the evidence for the realization of anisotropic structural features through the melt extrusion-based 3D printing which is a key development towards mimicking the anisotropic properties and hierarchical structures of biological materials in nature, such as human tissues.

\section{Response}

Dear Prof. Kirsten Severing,

We are submitting this original research communication entitled "Structural evolution of PCL during melt extrusion 3D printing" for your consideration. We confirm that this work is original and has not been published nor is it currently under consideration for publication elsewhere. 
do not add them to the cover letter.

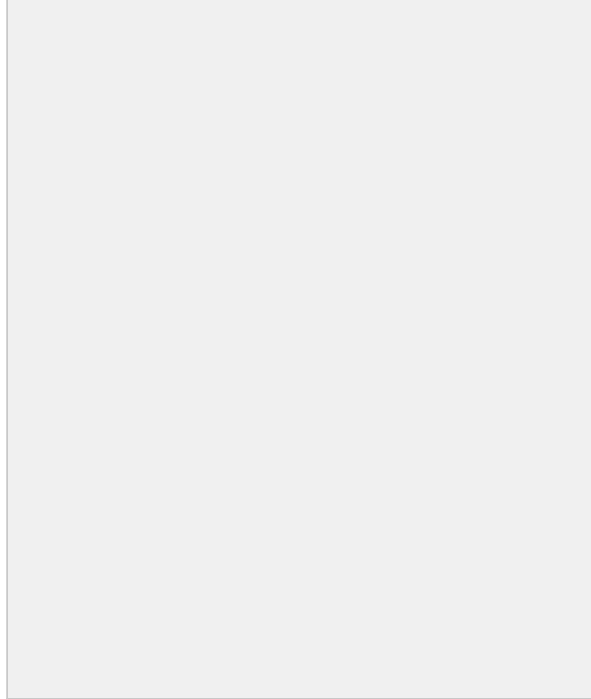

Do you or any of your co-authors have a conflict of interest to declare?
This communication is one of the first studies investigating the evolution of crystallinity and crystal orientation during melt extrusion 3D printing via in situ time-resolved synchrotron based $\mathrm{X}$-ray diffraction. We consider the results highly relevant to Macromolecular Rapid Communications readers and this communication timely due to its relevance for controlling the anisotropic structural features of 3D printed scaffolds for tissue engineering applications, which is a rapidly growing research field. We believe that Macromolecular Rapid Communications is the most suitable journal for this communication as it publishes high quality original research in polymer science, ranging from chemistry and physics of polymers in material science and life sciences. Also, the viewing time and average publication time are relatively short, good for fast publication of high priority results.

We would also like to emphasise that there are no conflicts of interest to disclose.

Sincerely,

Paulo Bartolo

Professor, School of Mechanical, Aerospace and Civil Engineering

University of Manchester

No. The authors declare no conflict of interest. 


\section{Structural evolution of PCL during melt extrusion 3D printinga}

Fengyuan Liu, Cian Vyas, Gowsihan Poologasundarampillai, Ian Pape, Sri Hinduja, Wajira Mirihanage*, Paulo Bartolo*

F. Liu. Author-One, C.Vyas. Author-Two, Prof. S. Hinduja. Author-Five, Prof. P.J. Bartolo. Author-Seven

School of Mechanical, Aerospace and Civil Engineering, University of Manchester, Oxford Road, Manchester, M13 9PL, United Kingdom.

Email: paulojorge.dasilvabartolo@manchester.ac.uk

Dr. G. Poologasundarampillai. Author-Three, Dr. W. Mirihanage. Author-Six

School of Materials, University of Manchester, Oxford Road, Manchester, M13 9PL, United Kingdom.

Dr. G. Poologasundarampillai. Author-Three

Research Complex at Harwell, Rutherford, Rutherford Appleton Laboratory, Harwell, OX11 OFA, United Kingdom.

I. Pape. Author-Four

Diamond Light Source, Didcot, Oxfordshire, OX11 0DE, United Kingdom.

Screw-assisted material extrusion technique has been developed for tissue engineering applications to produce scaffolds with well-defined multi-scale microstructural features and tailorable mechanical properties. In this study, in situ time-resolved synchrotron diffraction was employed to probe extrusion-based 3D printing of polycaprolactone (PCL) filaments. Time-resolved X-ray diffraction measurements revealed the progress of overall crystalline structural evolution of PCL during 3D printing. Particularly, in situ experimental observations provide strong evidence for the development of strong directionality of PCL crystals during the extrusion driven process. Results also show the evidence for the realization of anisotropic structural features through the melt extrusion-based 3D printing which is a key development towards mimicking the anisotropic properties and hierarchical structures of biological materials in nature, such as human tissues.

\footnotetext{
${ }^{a}$ Supporting Information ((bold)) is available online from the Wiley Online Library or from the author.
} 
1

2

3

4

5

6

7

8

10

11

12

13

14

15

16

17

18

19

20

21

22

23

24

25

26

27

28

29

30

31

32

33

34

35

36

37

38

39

40

41

42

43

44

45

46

47

48

49

50

51

52

53

54

55

56

57

58

59

60

61

62

63

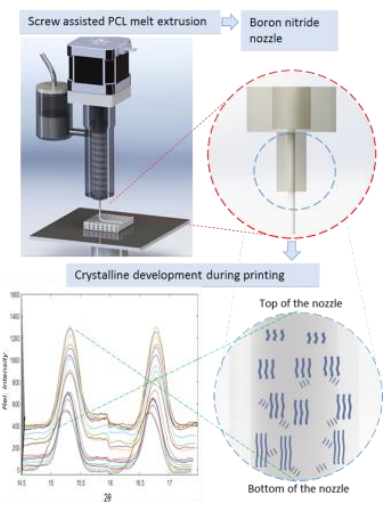

65 


\section{Introduction}

Regenerative medicine and tissue engineering are rapidly expanding fields that aim to repair, replace, and regenerate damaged cells, tissues, and organs ${ }^{[1-3]}$. Different methods have been explored including cell therapy, the use of scaffolds or tissue engineered scaffolds with cells and the use of cell-laden constructs ${ }^{[4]}$. However, the scaffold-based approach is very frequently used in tissue engineering. Scaffolds are three-dimensional biocompatible and biodegradable porous structures that provide substrates for cell attachment, differentiation and proliferation ${ }^{[5,6]}$. Scaffold design also requires the control of material properties, surface topography, chemistry and stiffness that significantly influence biological processes ${ }^{[7]}$. Furthermore, precise spatial positioning of different materials is required during the scaffold fabrication process. This has become possible with the development of additive biomanufacturing techniques which allow a wide variety of materials to be used including biological materials such as cells ${ }^{[8-10]}$. Different additive biomanufacturing strategies, such as powder bed fusion, stereolithography, extrusion-based and inkjet printing processes have been explored to produce scaffolds with precise control over material deposition and construct architecture for a variety of applications including bone, cartilage, nerve and skin ${ }^{[11-18]}$. Extrusion-based techniques are widely utilized due to the versatility and simplicity of the technique which enables a variety of materials to be processed, the ability to print in a cellfriendly environment as well as the feasibility for controlled high precision deposition. Different extrusion-based techniques have been developed including the use of pneumatic or mechanical assistance methods, utilizing a piston or a screw, to apply the extrusion force ${ }^{[19]}$. The screw-assisted method is ideal for producing scaffolds to realize bone regeneration as it allows precise deposition, high deposition velocity, improved material mixing, being able to manipulate materials with high viscosity ${ }^{[20]}$. 
PCL is one of the most commonly used synthetic polymers for tissue engineering and regenerative medicine applications ${ }^{[21-23]}$. This is due to the advantageous properties of PCL which make it suitable for tissue engineering applications. These include biocompatibility with it being utilized in many FDA approved devices, long-term biodegradability, ease of processing due to the low melting temperature $\left(\sim 58-60^{\circ} \mathrm{C}\right)$, suitable rheological and viscoelastic properties, and thermal stability ${ }^{[24-26]}$. PCL and PCL based composites have been explored in a range of applications in tissue engineering ${ }^{[27-30]}$. Different manufacturing techniques including additive biomanufacturing and conventional methods have been employed to fabricate PCL structures ${ }^{[31-33]}$. However, the effects of fabrication processing parameters have on the degree of crystallinity, crystallite size, and crystal orientation of the polymers, have not been widely researched. Crystallinity and crystal orientation ${ }^{[34]}$ of synthetic polymers are crucial since they can directly affect the scaffold properties having an effect on the cellular phenotype and cellular spreading ${ }^{[35]}$. In addition, the molecular weight of PCL influences the crystallization kinetics ${ }^{[36]}$, and also the bulk material stiffness ${ }^{[37]}$ can influence stem cell differentiation. However, structure evolution during filaments formation is rarely studied; probably because of its dynamic nature and the lack of experimental tools for such studies. In order to realize the full potential of $3 \mathrm{D}$ extrusion printing and in specific to engineer the properties of the printed constructs, it is essential to understand the development of microstructure during extrusion-based printing processes.

This paper presents one of the first studies to investigate the evolution of crystallinity and crystal orientation during melt extrusion via in situ time-resolved synchrotron X-ray diffraction (XRD).

\section{Experimental Section}

\section{1. $\quad$ Extrusion-based 3D printing process}


A new extrusion-based bioprinting machine, Plasma-assisted Bioextrusion System (PABS),

has been developed within the research group ${ }^{[38]}$ (Figure 1). The PABS system provides a novel approach for biomanufacturing and tissue engineering by combining screw-assisted extrusion, pressure-assisted extrusion, and plasma jetting. PABS not only enables a variety of materials, from cell-laden hydrogels to polymer composites, to be processed but also has the ability to modify the material surface through plasma jetting. This can influence surface properties such as chemical signaling, wettability, surface roughness and energy ${ }^{[39-41]}$ which can have an impact on material and biological interactions.

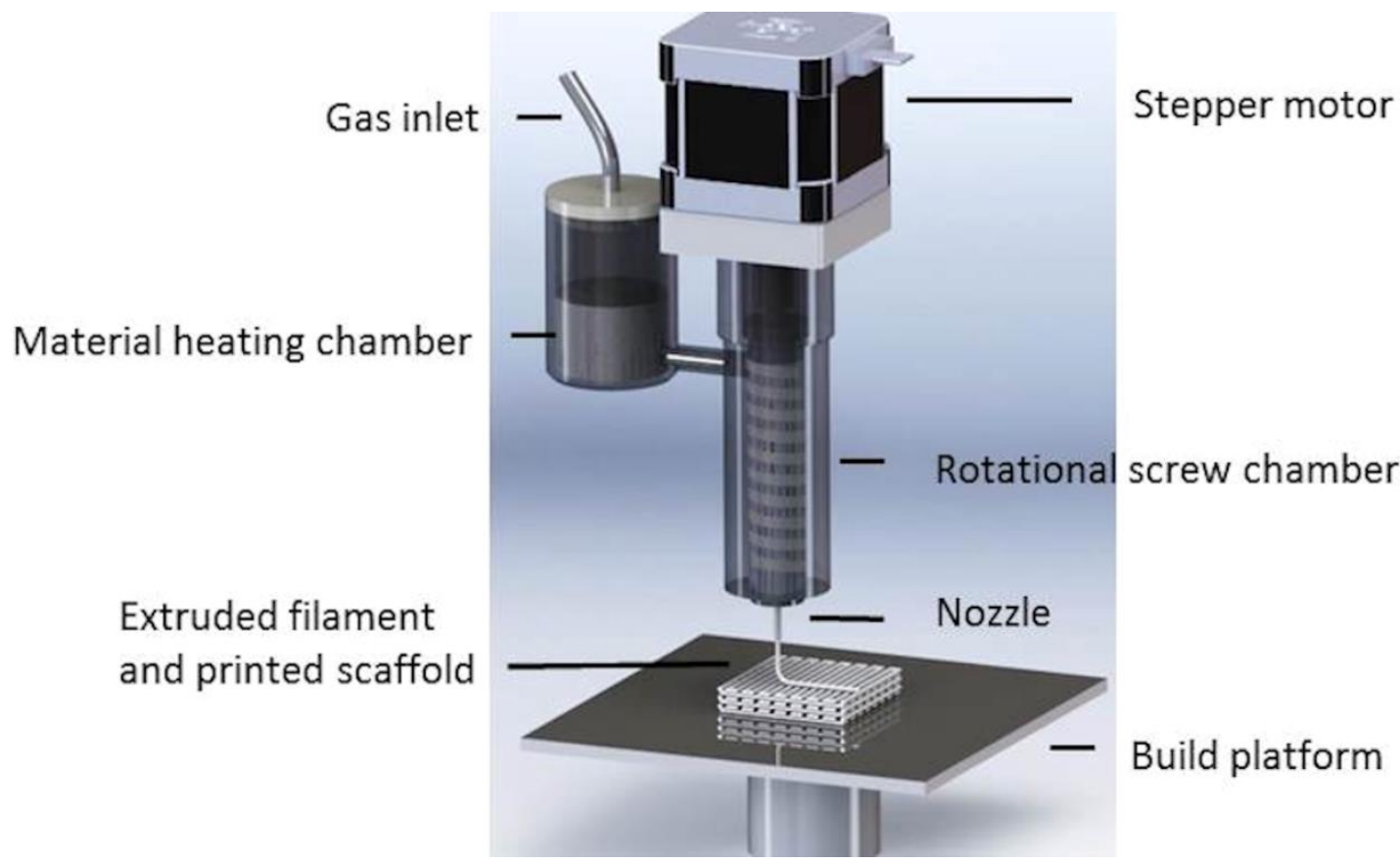

Figure 1. Schematic presentation of screw-assisted extrusion technique

In this study, only the screw-assisted extrusion system was utilized which consists of a material heating chamber and a rotational screw chamber. Materials are deposited in the chamber and the temperature rises above the melting temperature (Tm) to enable material flow. The material is delivered to the screw chamber through the application of compressed air (6 bar) from an externally connected compressor. Heating elements attached to the screw chamber maintain the required temperature, so that the material can be kept molten once delivered to the screw chamber. The rotational screw, driven by a stepper motor (Sanyo Denki, 
Japan), is employed to drive the material through the nozzle. A $0.5 \mathrm{~mm}$ inner diameter nozzle was utilized in this study forming PCL filaments with a circular cross section.

This extruder presents more precise deposition ability with high melting temperature (up to $\sim 200{ }^{\circ} \mathrm{C}$ ), faster extrusion speed (up to $20 \mathrm{rpm}$ ), improved material mixing, and greater suitability to manipulate materials with high viscosity. The screw rotational speed and melting temperature were kept at $10 \mathrm{rpm}$ and $110{ }^{\circ} \mathrm{C}$. This setting yielded a printed speed of PCL filament at $0.1 \mathrm{mms}^{-1}$.

\subsection{PCL Microstructure}

In this study, PCL $\left(\mathrm{CAPA}^{\circledR} 6500, \mathrm{Mw}=50,000 \mathrm{~g} / \mathrm{mol}\right)$ purchased from Perstorp (Cheshire, United Kingdom) in the form of $3 \mathrm{~mm}$ pellets was used. PCL is a semi-crystalline polymer with a density of $1.1 \mathrm{~g} / \mathrm{cm}^{3}$, a melting temperature between $58-60{ }^{\circ} \mathrm{C}$, and a glass transition temperature of $-60^{\circ} \mathrm{C}$.

The PCL pellets and printed filaments were sliced using a Leica CM3050 S cryostat to obtain samples of $10 \mu \mathrm{m}$ and $5 \mu \mathrm{m}$ thickness respectively, for ex situ optical microscopy. It is important to note that the samples must be thin enough for birefringent spots to be observed as a measurement of the degree of crystallinity, in which the single crystal formula can be applied ${ }^{[42]}$. Figure 2 shows the microstructures of both as received pellets and filament samples obtained using polarizing light microscope (Olympus BH2-UMA). In the PCL pellet, birefringent spherulitic structures were observed which exhibited the Maltese cross typical of polymers. It is apparent that the size of the sperulites in the PCL pellet were reduced dramatically from a maximum radius of $\sim 40 \mu \mathrm{m}$ to less than $4 \mu \mathrm{m}$ for the printed PCL filaments with an increasing number of spherulites, suggesting smaller crystals. These microscopy images do not allow the observation of any preference in crystal orientation 
to perceive how orientated crystal arrangement evolves during the extrusion process.
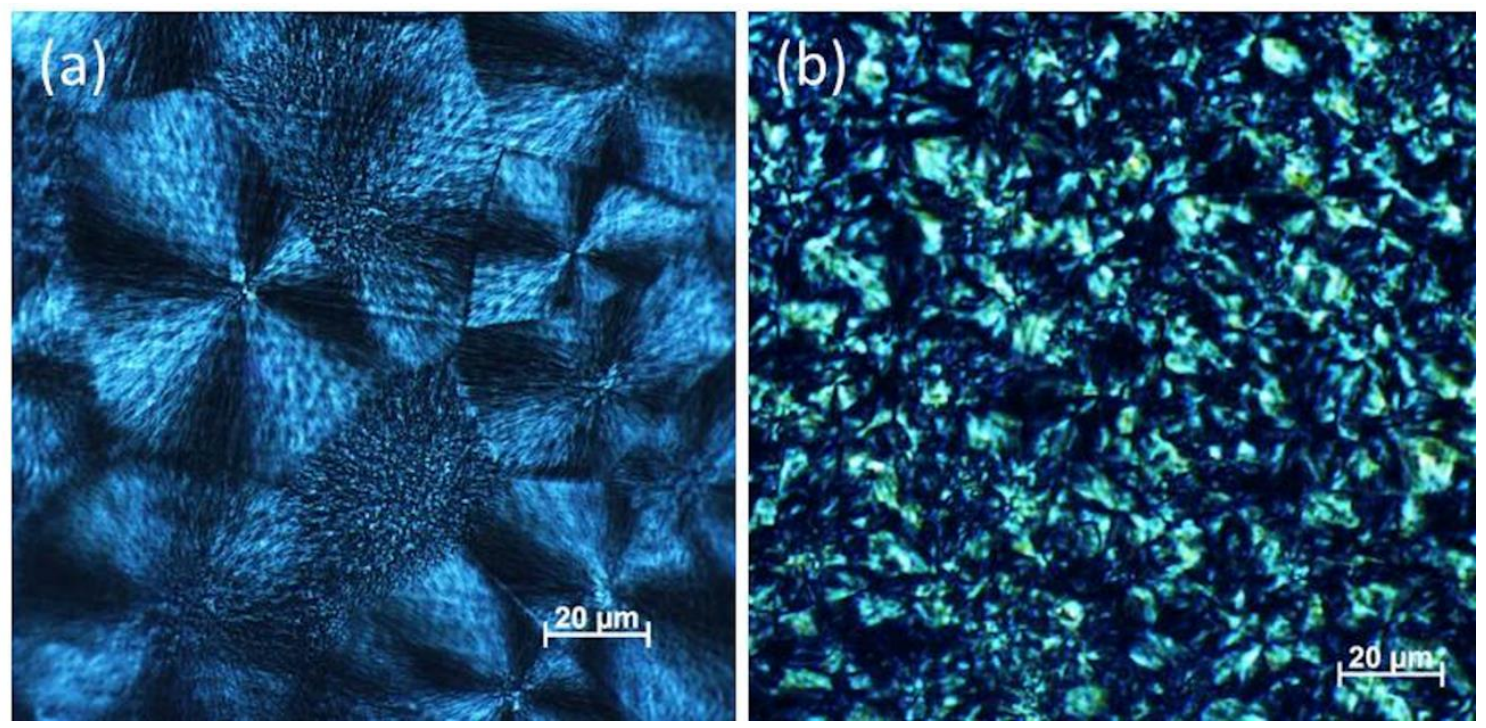

Figure 2. Microstructures of (a) PCL raw pellet and (b) extruded filament (melting temperature of $110{ }^{\circ} \mathrm{C}$ and screw rotational speed of $10 \mathrm{rpm}$ ) obtained using polarized light microscopy.

\subsection{In situ synchrotron $X$-ray diffraction}

The evolution of the crystalline structure of PCL during extrusion was examined by using in situ synchrotron wide angle X-ray scattering, which is also referred to as X-ray Diffraction (XRD) at the Diamond Light Source (Harwell, UK) on beamline B16. The beamline's multilayer monochromator delivered X-ray photons with a nominal $17.98 \mathrm{keV}(\Delta \mathrm{E}=200 \mathrm{eV})$ energy. The beamline's slits were adjusted to a defined $200 \times 200 \mu \mathrm{m}^{2}$ beam size for this study. The experimental setup involved simultaneous in situ transmission of XRD and radiographic images (Figure 3). However, radiographic imaging was unable to deliver sustained real space information related to the processing of PCL, and consequently is not discussed in this paper. For in situ XRD data collection, a Pilatus ${ }^{\circledR} 300 \mathrm{~K}$ area detector was mounted at $45^{\circ}$ to the beam direction. XRD patterns were collected at $1 \mathrm{~Hz}$ frequency $(0.9 \mathrm{~s}$ exposure time). The distance from the sample to the detector was $266 \mathrm{~mm}$. A boron nitride extrusion nozzle with a $1.5 \mathrm{~mm}$ and $0.5 \mathrm{~mm}$ external and internal diameter, respectively, was fabricated and used in this research in order to facilitate adequate X-ray penetration. To track 
the structural evolution of a pre-selected volume of PCL, the counter motion of the entire

(a)

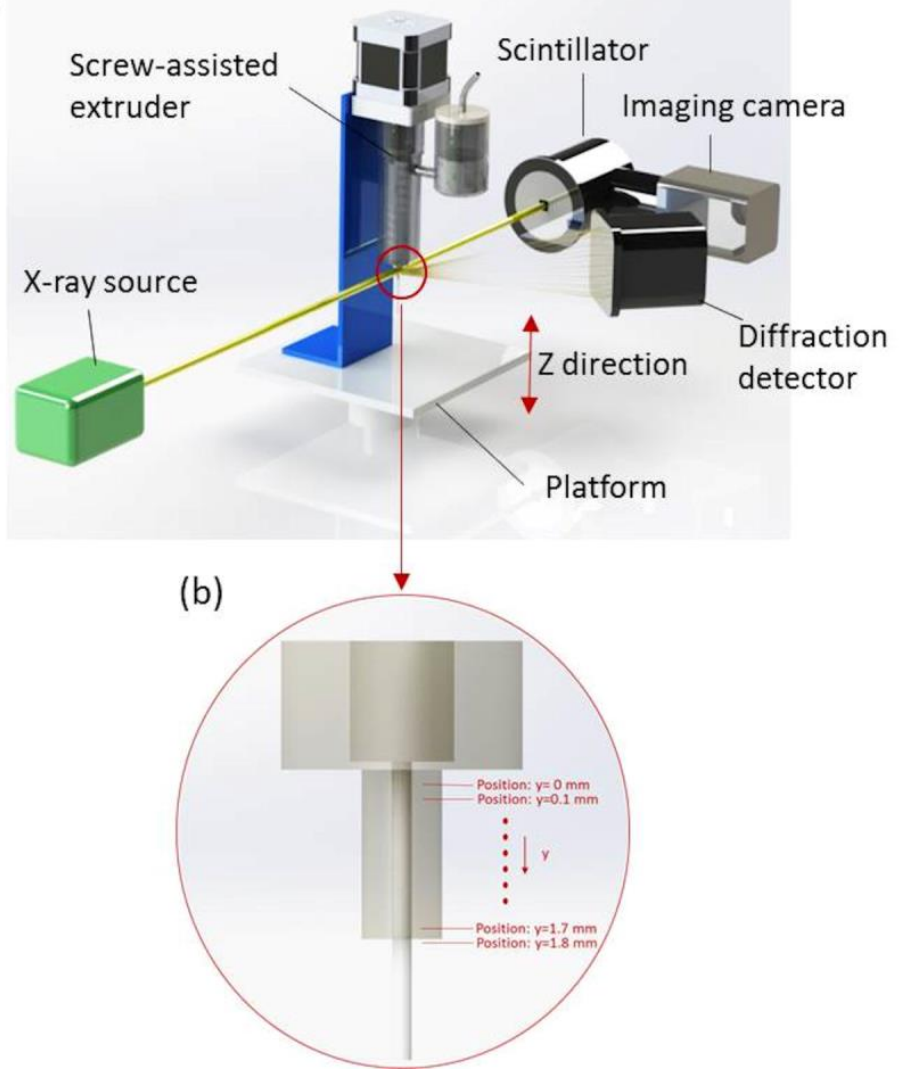

Figure 3. (a) schematic setup of in situ XRD experiment; (b) schematic presentation of nozzle tip and 19 XRD diffraction positions accessed during the experiments (positions: $y=0-1.8$ $\mathrm{mm})$. 
Data processing and background reduction was carried out using a bespoke MATLAB program, which included the conversion of diffraction patterns recorded at $45^{\circ}$ angle to the beam direction into observations in the plane orthogonal to the incident $\mathrm{X}$-ray beam employing a rotational matrix for each detector pixel. The pixel intensities were subjected to other standard image/X-ray data processing operations such as radial or azimuthal integrations and peak fitting.

\section{Results and Discussion}

\subsection{Crystal orientation}

Selected in situ X-ray diffraction patterns correspond to positions $\mathrm{y}=0-1.8 \mathrm{~mm}$ (time: $0-18 \mathrm{~s}$ ) are illustrated in Figure 4a and in Movie 1 in the supporting information.

The evolution of the PCL XRD patterns reflects the crystal structural changes that PCL undergoes during the printing process (Figure 4a) The evolution of low order (020) and (120) reflections from the orthorhombic PCL structure was more prominent in the background corrected images. At the uppermost diffracted positions in the nozzle (ie. positions: $y=0-0.7$ $\mathrm{mm})$, a discrete region of each Debye-Scherer ring was observed for (020) and (120) suggesting crystalline features with a directional alignment. Once the material is pushed through the nozzle (ie. positions: $y=0.8-1.2 \mathrm{~mm}$ ), the intensity of the arcs increases while the arc length increases over wider azimuthal angle. At the exit end of the nozzle (ie. positions: $\mathrm{y}=1.4-1.8 \mathrm{~mm}$ ), the length of the arcs was further increased. Even though the extended arc length of both reflections was visible, initial sections of the arcs appeared brighter compare to the extended regions. The observation indicates the continuation of anisotropic structural preference ${ }^{[43]}$, although it has been reduced by some extent when compared to the initial levels. 


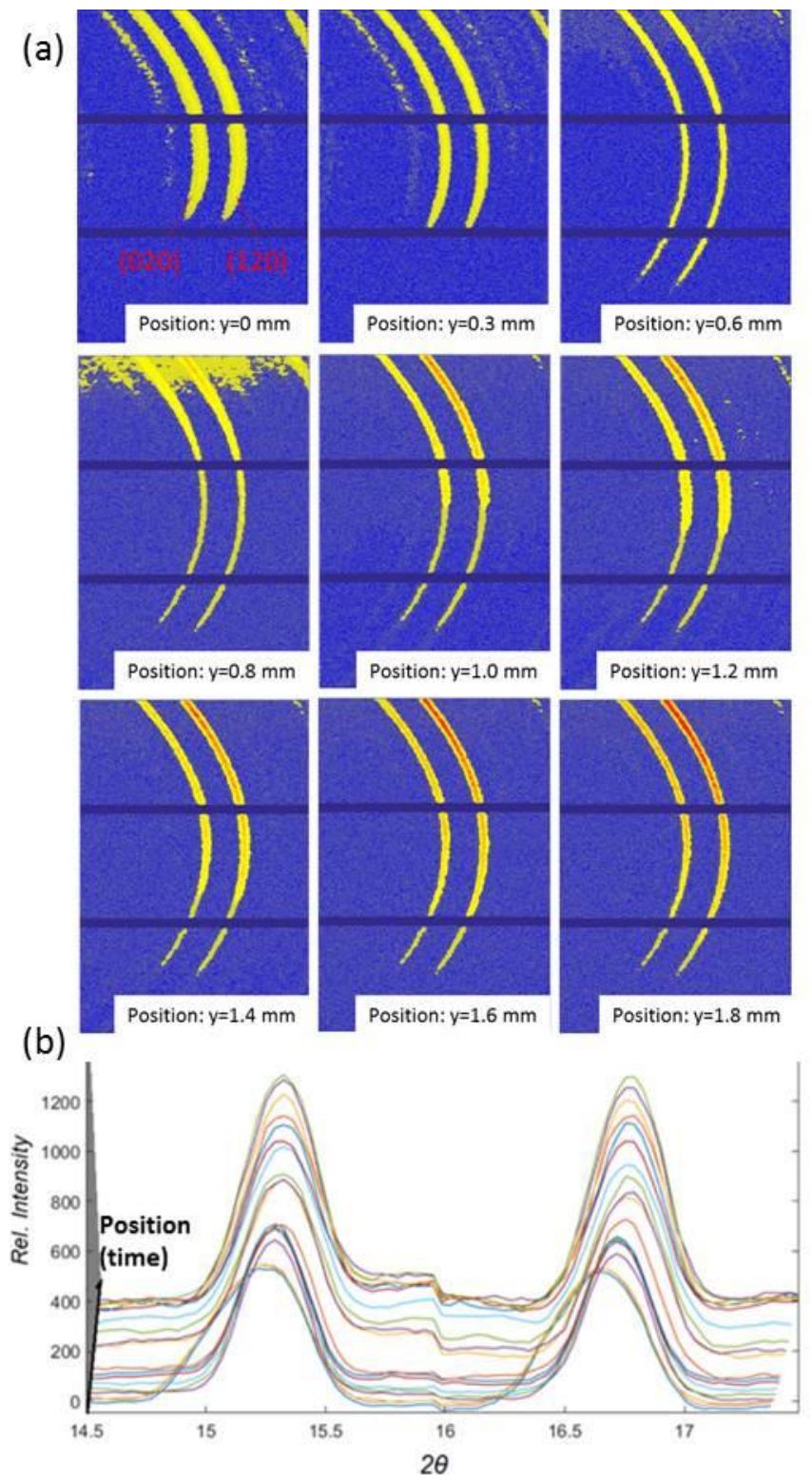

Figure 4. (a) Selected in situ XRD patterns from the same volume of PCL as it flows down inside the nozzle during extrusion 3D printing. (b) Refined XRD intensity evolution for (020) at $2 \theta=\sim 15.3$ and (120) at $2 \theta=\sim 16.8$ reflections as a function of positions in the nozzle. Lower curve corresponds to position $\mathrm{y}=0 \mathrm{~mm}$, upper curve corresponds to position $\mathrm{y}=1.8 \mathrm{~mm}$.

\subsection{Crystal volume fraction}

To further elucidate the evolution of crystalline detail during extrusion through the nozzle, the XRD patterns obtained from the 2D area detector (Figure 4a) were transformed to a conventional one-dimensional (1D) XRD representation as shown in Figure $4 \mathbf{b}$. The transverse axis was recognized as the Bragg angle (20). Figure $4 \mathbf{b}$ shows the scattering 
intensity evolution of (020) and (120) reflections for all 18 linear spatial positions as the proportional to the volume fraction of the crystals ${ }^{[44]}$, so that the increasing integrated intensity from position $\mathrm{y}=0-1.8 \mathrm{~mm}$ in the nozzle indicates that the volume of the crystals is increasing during the extrusion of PCL. As shown in Figure $\mathbf{4 b}$, the integrated peak intensity increases as the material flows through the nozzle.

Two main characteristics can be identified from the XRD patterns at the top of the nozzle. At position $\mathrm{y}=0 \mathrm{~mm}$, where the time is taken as $0.0 \mathrm{~s}$, an already crystalized volume can be recognized. This is attributed to the small amount of PCL crystal nucleation taking place before the polymer melt enters the nozzle. Although the temperature was expected to be maintained in both the material heating chamber and screw chamber during the process, as the material flows into the nozzle tip, a temperature gradient is unavoidable between the extruder head and the nozzle. Thus, the PCL melt can be partially undercooled before it reaches the extruder nozzle. It was shown that nucleation and partial crystallization in the PCL were enhanced by substantial undercooling ${ }^{[45]}$ as well as the short step stress ${ }^{[46]}$. These are the two potential mechanisms that mostly can be operated in the melt extrusion-based 3D printer. Particularly, the short step stress can be introduced due to the rapid cross sectional change associated when material enters the nozzle from the chamber. The other important feature to be noted is that the crystal orientation was uniform at this stage. As previously reported the crystallographic c axis of polymer crystals tends to be oriented perpendicular to the extrusion direction, and the preferred crystal orientation (fiber axis) is parallel to the drawing direction (Figure 5). For further details of crystallographic orientation of polymers readers are referred to ref. [47]. At the beginning arc length is shorter due to crystals organized parallel to extrusion direction, the increased intensity of the initial arc region (azimuthal angles) indicate increased crystal volume faction oriented in that direction. However, the extension of arc length covering wider azimuthal angle with relatively low intensity at the bottom of the 
nozzle suggests a small crystal volume fraction is oriented in a direction deviated from the increasing manner, but at a gradually reducing rate, along the nozzle distance (relative to the final measurement at $\mathrm{y}=1.8 \mathrm{~mm}$ where crystal faction taken as one). It also presents a schematic presentation of the orientation development during the extrusion of PCL through the nozzle. The results suggest that the shear field generated during printing enhances the formation of oriented crystals in the polymer melts, which is consistent with the literature ${ }^{[48]}$. The actual flow conditions within the extruder nozzle can be potentially explained by a small boundary layer within the nozzle that introduces a negligible radial velocity profile. However, detailed modelling is currently not available to investigate this hypothesis. Furthermore, the rapid undercooling, with relatively high temperature gradient within the nozzle, appears to be the dominant influencing factor in crystallization in this specific 3D printing extrusion-based process.

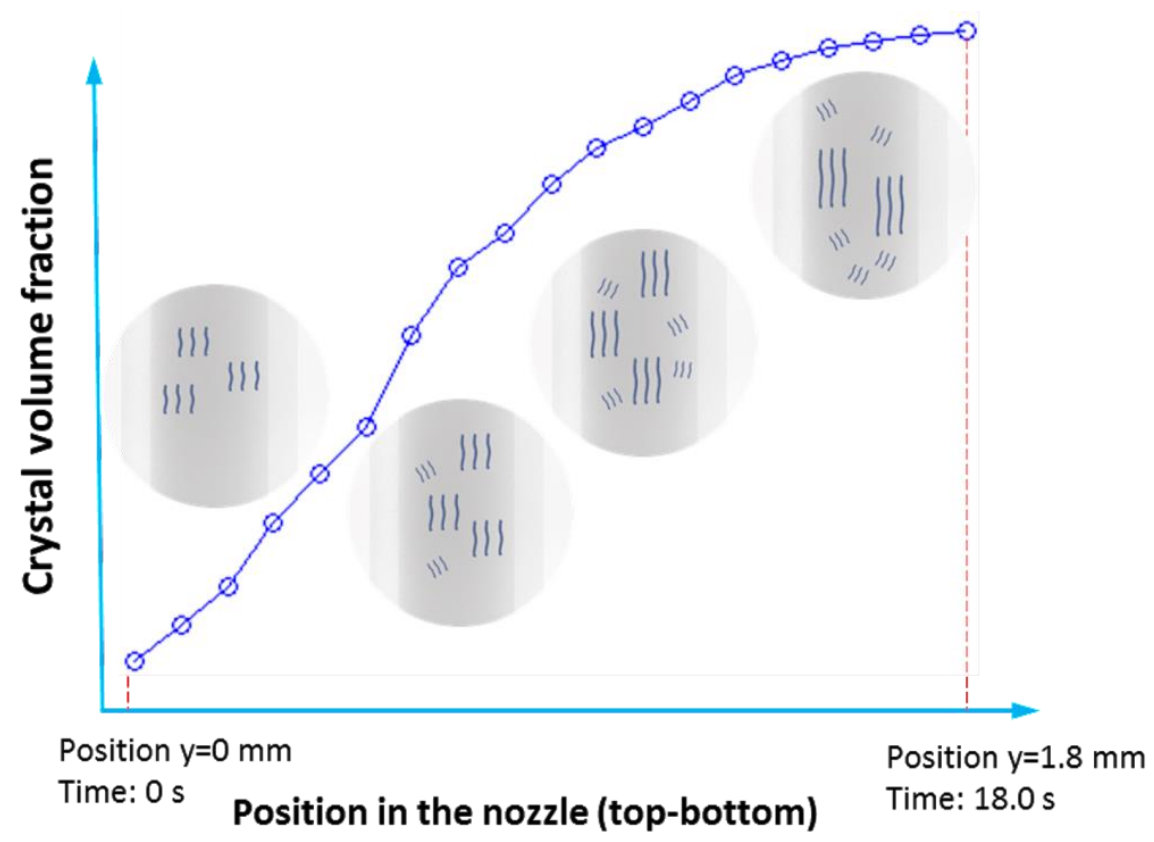

Figure 5. Schematic representation of microstructures and crystal volume fraction from top to bottom positions in the nozzle. Crystal volume fraction presents an increasing trend with the material extruding from top to the bot-tom in the nozzle tip. The majority of the crystal 


\section{fraction grows along the axial direction whilst a smaller fraction is observed to grow in other}

\section{directions with increasing position in the nozzle.}

As seen in Figure $\mathbf{4 b}$, a slight but gradual shift in $2 \theta$ diffraction of (020) and (120) while extruding through the nozzle is typically attributed to thermal contraction. However, considering the reported thermal expansion coefficient of PCL with decreasing temperature along the nozzle, a $2 \theta$ change is more than the corresponding thermal shrinkage. The difference can be attributed to the potential compressive strain present in the material. Presence of shear stresses and compressive stress are common phenomena during extrusion processes. Such stress/strain effects must be considered in the future through the use of a precise thermal model for a detailed and quantitative analysis of the extruder nozzle, hence no attempt is made to analyze and present the quantitative figures or stress and stress driven interactions here.

According to the observations, crystallization occurs as the material moves through the nozzle to a substantial level. However, once the material is ejected from the nozzle, further development/evolution of crystallinity is anticipated during subsequent elongation of the polymer because of the printer head stretching the filament during the prescribed $x-y$ movement typical of the 3D printing process. Additionally, as the polymer cools further increase of crystal fraction may occur. Future work includes the investigation of crystallization under different processing parameters and PCL based composites including bioactive ceramics such as hydroxyapatite in order to understand multiscale structural formation in the presence of crystal modifying agents, in addition to the development of quantitative modelling capabilities to simulate the process. This will enable the development of bespoke engineered materials for tissue engineering applications, such as human bone regeneration.

\section{Conclusions}


This contribution provides a unique real time in situ XRD characterization of crystal formation in PCL filaments during melt extrusion-based 3D printing process. The presence of crystalline anisotropy was identified and the majority of crystals were oriented in the direction of material flow. In situ time-resolved characterization shows that there is a gradual, but not complete, reduction of directionality with the increasing crystal fraction of the PCL crystals during flow through the extrusion nozzle. In addition, experimental and data analysis procedures presented here demonstrate a novel example and a promising method to employ real time synchrotron XRD as a tool for probing additive biomanufacturing processes.

\section{Acknowledgements}

The authors wish to acknowledge the funding provided by the Engineering and Physical Sciences Research Council (EPSRC) and Medical Research Council (MRC) Centre for Doctoral Training in Regenerative Medicine (EP/L014904/1). Diamond Light Source is acknowledged for granting beamtime (MT14877-1) and authors are grateful to Dr. Kawal Sawhney for his advice on experimental design.

Technical support from Andrew Malandain (DLS), Kenneth Gyves and Marie O’Brien (UoM) is also acknowledged and greatly appreciated.

\section{Keywords}

Additive biomanufacturing, crystallization, in situ X-ray diffraction, screw-assisted melt extrusion

\section{References}

[1] R. F. Pereira, A. Sousa, C. C. Barrias, A. Bayat, P. L. Granja, P. J. Bártolo, Biomanufacturing Reviews 2017, 2, 1.

[2] C. Vyas, R. Pereira, B. Huang, F. Liu, W. Wang, P. Bartolo, Current Opinion in Biomedical Engineering 2017, 2, 1.

[3] W. Wang, G. Caetano, W. Ambler, J. Blaker, M. Frade, P. Mandal, C. Diver, P. Bártolo, Materials 2016, 9, 992. 
[4] R. F. Pereira, P. J. Bártolo, Journal of Applied Polymer Science 2015, 132, n/a. Kaplan, F. G. Omenetto, Nat Nano 2017, 12, 474.

[6] X.-Y. Yang, L.-H. Chen, Y. Li, J. C. Rooke, C. Sanchez, B.-L. Su, Chemical Society Reviews 2017, 46, 481.

[7] Q. Zhang, S. Lin, Q. Li, D. Zhao, X. Cai, "Cellular Response to Surface Topography and Substrate Stiffness", in Cartilage Regeneration, Y. Lin, Ed., Springer International Publishing, Cham, 2017, p. 41.

[8] S.-S. D. Carter, P. F. Costa, C. Vaquette, S. Ivanovski, D. W. Hutmacher, J. Malda, Annals of biomedical engineering 2017, 45, 12.

[9] M. P. Chhaya, E. R. Balmayor, D. W. Hutmacher, J.-T. Schantz, Scientific Reports 2016, $6,28030$.

[10] X.-Y. Zhang, G. Fang, J. Zhou, Materials 2017, 10, 50.

[11] S. F. S. Shirazi, S. Gharehkhani, M. Mehrali, H. Yarmand, H. S. C. Metselaar, N. A. Kadri, N. A. A. Osman, Science and technology of advanced materials 2015, 16, 033502.

[12] X. Cui, G. Gao, T. Yonezawa, G. Dai, Journal of visualized experiments: JoVE 2014.

[13] C. Murphy, K. Kolan, W. Li, J. Semon, D. Day, M. Leu, International Journal of Bioprinting 2017, 3, 1 .

[14] D. Ronca, F. Langella, M. Chierchia, U. D’Amora, T. Russo, M. Domingos, A. Gloria, P. Bartolo, L. Ambrosio, Procedia CIRP 2016, 49, 51.

[15] S. Vega, M. Kwon, J. Burdick, European cells \& materials 2017, 33, 59.

[16] J. Ribeiro, T. Pereira, I. Amorim, A. R. Caseiro, M. A. Lopes, J. Lima, A. Gartner, J. D. Santos, P. J. Bártolo, J. M. Rodrigues, International journal of medical sciences 2014, 11, 979. [17] C.Cyan, G. Poologasundarampillai, J.Hoyland, P. Bartolo, "3D Printing of Biocomposites for Osteochondral Tissue Engineering", in Biomedical Composites, Elsevier, 2017, p.261.

[18] L. Shor, S. Güçeri, R. Chang, J. Gordon, Q. Kang, L. Hartsock, Y. An, W. Sun, Biofabrication 2009, 1, 015003.

[19] S. V. Murphy, A. Atala, Nature biotechnology 2014, 32, 773.

[20] L. G. Zhang, J. P. Fisher, K. Leong, "3D Bioprinting and nanotechnology in tissue engineering and regenerative medicine", Academic Press, 2015.

[21] H. Rashidi, J. Yang, K. M. Shakesheff, Biomaterials Science 2014, 2, 1318.

[22] P. Carter, S. M. Rahman, N. Bhattarai, Journal of Biomaterials science, Polymer edition 2016, 27, 692.

[23] N. Thadavirul, P. Pavasant, P. Supaphol, Journal of Biomedical Materials Research Part A 2014, 102, 3379.

[24] B. Guo, P. X. Ma, Science China Chemistry 2014, 57, 490.

[25] M. Okamoto, B. John, Progress in Polymer Science 2013, 38, 1487.

[26] M. A. Woodruff, D. W. Hutmacher, Progress in Polymer Science 2010, 35, 1217.

[27] T. S. Plivelic, S. N. Cassu, M. do Carmo Gonçalves, I. L. Torriani, Macromolecules 2007, 40, 253.

[28] M. S. Kamath, S. S. Ahmed, M. Dhanasekaran, S. W. Santosh, International journal of nanomedicine 2014, 9, 183.

[29] S. Sayyar, E. Murray, B. C. Thompson, S. Gambhir, D. L. Officer, G. G. Wallace, Carbon 2013, 52, 296.

[30] R. Augustine, S. K. Nethi, N. Kalarikkal, S. Thomas, C. R. Patra, Journal of Materials Chemistry B 2017.

[31] O. Bas, E. M. De-Juan-Pardo, C. Meinert, D. D’Angella, J. G. Baldwin, L. J. Bray, R. M. Wellard, S. Kollmannsberger, E. Rank, C. Werner, Biofabrication 2017, 9, 025014.

[32] B. T. Vinson, S. C. Sklare, D. B. Chrisey, Current Opinion in Biomedical Engineering 2017. 
[33] T. Guo, T. R. Holzberg, C. G. Lim, F. Gao, A. Gargava, J. E. Trachtenberg, A. G. Mikos, J. P. Fisher, Biofabrication 2017, 9, 024101.

[34] J. Liu, D. Y. Lin, B. Wei, D. C. Martin, Polymer 2017, 118, 143.

[35] M. I. Sabir, X. Xu, L. Li, Journal of materials science 2009, 44, 5713.

[36] B. Zhou, W.-N. He, X.-Y. Jiang, Z.-Z. Tong, J.-T. Xu, Z.-Q. Fan, Composites Science and Technology 2014, 93, 23.

[37] W. Hendrikson, J. Rouwkema, C. Van Blitterswijk, L. Moroni, RSC advances 2015, 5, 54510.

[38] F. Liu, S. Hinduja, P. Bartolo, Procedia CIRP 2017, 65, 213.

[39] D. S. Syromotina, R. A. Surmenev, M. A. Surmeneva, A. Boyandin, E. Nikolaeva, O. Prymak, M. Epple, M. Ulbricht, C. Oehr, T. Volova, Materials Science and Engineering: $C$ 2016, 62, 450.

[40] F. Intranuovo, R. Gristina, F. Brun, S. Mohammadi, G. Ceccone, E. Sardella, F. Rossi, G. Tromba, P. Favia, Plasma Processes and Polymers 2014, 11, 184.

[41] F. Wen, C. C. S. Lau, J. Lim, Y. Liao, S. H. Teoh, M. S. K. Chong, "Surface Modification of Tissue Engineering Scaffolds", in Polymeric Biomaterials for Tissue Regeneration, Springer, 2016, p. 123.

[42] V. Speranza, A. Sorrentino, F. De Santis, R. Pantani, The Scientific World Journal 2014, 2014.

[43] X. Wang, H. Zhao, L.-S. Turng, Q. Li, Industrial \& Engineering Chemistry Research 2013, 52, 4939.

[44] W. Zachariasen, Acta Crystallographica 1967, 23, 558.

[45] A. Wurm, E. Zhuravlev, K. Eckstein, D. Jehnichen, D. Pospiech, R. Androsch, B. Wunderlich, C. Schick, Macromolecules 2012, 45, 3816.

[46] G. Floudas, L. Hilliou, D. Lellinger, I. Alig, Macromolecules 2000, 33, 6466.

[47] P. Lindenmeyer, S. Lustig, Journal of Applied Polymer Science 1965, 9, 227.

[48] R. H. Somani, B. S. Hsiao, A. Nogales, H. Fruitwala, S. Srinivas, A. H. Tsou, Macromolecules 2001, 34, 5902. 
Click here to access/download Supporting Information Supporting information.docx 
Click here to access/download Supporting Information Video1.avi 
Click here to access/download Production Data figure of abstract.tif 
Click here to access/download

Production Data

Picture1.tif 
Click here to access/download

Production Data

Picture2.tif 
Click here to access/download

Production Data

Picture3.tif 
Click here to access/download

Production Data

Picture4.tif 
Click here to access/download

Production Data

Picture5.tif 
Click here to access/download Production Data summery.docx 
Click here to access/download

Production Data

Table of Content.docx 
Click here to access/download

Production Data TOC.tif 